\title{
Effectiveness of perineural and intravenous dexamethasone added to bupivacaine for transversus abdominis plane block in post-cesarean delivery pain control: a prospective cohort study, 2019
}

Bedru Jemal ( $\sim$ bedruanst@gmail.com )

Dilla University College of Health Sciences https://orcid.org/0000-0002-4194-4724

Abebayehu Zemedkun

Dilla University College of Health Sciences

Wosenyeleh Admasu

Addis Ababa University School of Medicine

Sileshi Abiy

Addis Ababa University School of Medicine

Simeneh Molla

Dilla University

Hailemariam Mulugeta

Dilla University

Research article

Keywords: TAP block, intravenous dexamethasone, perineural dexamethasone

Posted Date: March 4th, 2020

DOI: https://doi.org/10.21203/rs.3.rs-16055/v1

License: (c) (i) This work is licensed under a Creative Commons Attribution 4.0 International License.

Read Full License 


\section{Abstract}

Background Caesarean delivery (CD) has been one of the most frequently performed major surgical interventions, and causes severe postoperative pain. Abdominal field blocks like TAP block are mostly preferred as post-operative analgesia for this operation. Using different adjuvants like dexamethasone by different routes increases the quality and duration of block and maximizes patient satisfaction. The objective of this study was to compare the effectiveness between perineural and intravenous dexamethasone as an adjuvant on bilateral transversus abdominis plane block for post cesarean delivery pain control.

Methodology An institutional based prospective cohort study was conducted on 87 patients. Study participants were selected by systematic random sampling technique. Data collection methods include preoperative chart review, intraoperative observation and postoperative patient interview at 4 th , 6 th , 8 th , 12 th and 24 th hours. Sociodemographic variable were analyzed by ANOVA and chi square test. Kruskal Wallis with post hoc analysis was used to compare post-operative severity of pain score and cumulative analgesic consumption. Time to first analgesic request was analyzed using Kaplan Meier survival analysis with log rank. Categorical variable were analyzed by chi square.

Result Time to first analgesic request was significantly longer in TAP-IVD and TAP-PD compared to TAP alone $(p<0.05)$. The postoperative NRS score at rest and on coughing were significantly lowered in TAPPD and TAP-IVD group compared to TAP alone group $(\mathrm{p}<0.05)$. The total analgesic consumption in the first 24h was significantly lower in TAP-IVD and TAP-PD group compared to TAP alone group $(p<0.05)$.

Conclusion and recommendation dexamethasone $8 \mathrm{mg}$ both intravenously and perineurally is effective adjuvant to bupivacaine on bilateral TAP block with prolonged and potent analgesia and reduced analgesic consumption. Based on our finding we recommend use of dexamethasone as an adjuvant to bupivacaine on bilateral transversus abdominis plane block.

\section{Introduction}

Cesarean section is an operative technique by which a fetus is delivered through an abdominal and uterine incision. When adequately indicated it can prevent poor obstetric outcomes and be a life-saving procedure for both the mother and the fetus(1)

Cesarean section is the most commonly performed operation in the world. The WHO report shows the global average caesarean delivery (CD) rate increased from 12.4-18.6\% in the year 1990-2014 (2). Ethiopian demographic health survey found that the Addis Ababa CD rate was $22 \%$ which is higher than the WHO recommendations as treatments of pregnancy (3).

Managing post-operative pain has importance like decreasing hospital stay and associated costs, decreasing hospital acquired infections, allowing early mobilization, facilitating mother to fetal bonding, reducing thrombo-embolic events and increasing patient satisfaction. Inadequate postoperative pain 
relief after Caesarean delivery can negatively impact ambulation, breastfeeding, even maternal bonding and leads to chronic pain syndromes and poor quality of life (4-7).

Dexamethasone is easily affordable, accessible, cheap and safe. So that, both intravenously and perineurally has been used as adjuvant to bupivacaine on tansversus abdominis plane block in the study area to enhance quality and duration of analgesia.

To date, it is unclear whether the perineural administration confers advantages over the IV administration of this drug $(8,9)$. The result of our study may show possible analgesic modalities for postoperative pain control in caesarean section patients in resource limited set-ups like ours where alternative drugs and ultrasound is not available and almost all regional blocks are performed with blind technique. The primary objective of this study was to compare effectiveness between perineural and intravenous dexamethasone as an adjuvant on bilateral transversus abdominis plane block for post cesarean delivery pain control for mothers operated under spinal anesthesia. The secondary objective was to compare incidence of complications (nausea, vomiting and shivering) between the groups.

\section{Methods And Materials}

An institutional-based prospective cohort study was employed from January 1 to April 30, 2019 in Mahatma Gandhi and Empress Zewditu Memorial Hospitals, the public hospitals in Addis Ababa, capital of Ethiopia. Ethical clearance was obtained from the Addis Ababa university institutional review board before the start of the study. Official support letter was written to the Hospitals and permission for data collection was sought from the responsible authorities. Verbal as well as written informed consent was obtained from each participant. All ASA I and II parturients who underwent elective caesarean delivery under spinal anaesthesia were included in this study. Parturients with preeclampsia, eclampsia, history of medical illness, history of chronic opioid use, diabetes mellitus, use of other adjuvant, use of adjuvants for spinal anesthesia, bleeding abnormality and patients with $\mathrm{BMI}>30 \mathrm{~kg} / \mathrm{m} 2$ were excluded from the study.

The protocol and routine procedures before, during and after CS at the study site were as follows:

On arrival of the patients to the operative theater, and after application of the routine hospital monitoring protocol, HR, noninvasive blood pressure, and SPO2 has been recorded before institution of spinal anesthesia, then all patient received spinal anesthesia with $2-3 \mathrm{ml}$ of $0.5 \%$ bupivacaine (according to the height of the patient) using 25 or 26-Gauge spinal needle based on the responsible anesthetists preference. After this, all patients were repositioned in supine position and level of sensory and degree of motor block was assessed. Then, the necessary Intraoperative data was recorded. We the investigators did not participate in the perioperative patient management and post-operative pain management option.

In study hospital postoperative pain management for CS are done by either bilateral TAP block with bupivacaine alone (40 $\mathrm{ml}$ (20 $\mathrm{ml}$ each side), $0.25 \%)$, with IV dexamethasone (8 $\mathrm{mg})$ or perineural 
dexamethasone (8 $\mathrm{mg})$ depending the decision of anesthetist in charge. The block was performed by using a standard land mark technique immediately after skin closure.

Since randomized control trial (RCT) was not yet allowed in our university, the patients were not randomized, rather patients were classified as TAP-alone $(n=29)$, TAP-IVD $(n=29)$ and TAP-PD $(n=29)$ based on the responsible anesthetists decision at the end of skin closure. TAP-alone group were considered as control group

Under aseptic technique, after identifying lumbar triangle of Petit as an access point to the neurofascial plane, TAP block has been performed by Msc anesthesia trainee or staff Msc on duty with the patient lying in supine position. Following aseptic preparation of the skin, $22 \mathrm{G}$ blunted needle was advanced perpendicular to skin $2 \mathrm{~cm}$ above anterior superior iliac spine in triangle of petit, as the external oblique muscle is pierced a characteristic 'click' or 'pop' was felt and as the needle was advanced further a second 'click' was felt as the internal oblique muscle is pierced then after care full aspiration $20 \mathrm{ml}$ of $0.25 \%$ bupivacaine (for one side) was injected in the facial plane (TAP-Alone).

Eight (8) mg Dexamethasone was given either mixed with bupivacaine or IV immediately when the block is performed (for TAP-PD and TAP-IVD respectively).

The same procedure has been performed at the contra lateral side and the patients were transferred to the obstetric recovery ward after the procedure

Postoperative pain was assessed in all groups using a NRS score. The scale consists of horizontal lines ranging from 0 (no pain) to 10 (worst imaginable pain). Patients were asked to report their pain based on 11 point NRS score. The pain intensity was rated as no pain (NRS: 0), mild (NRS: $1-3$ ), moderate (NRS: 4-6), and severe (NRS: 7-10). The first NRS score was recorded at 4 th $\mathrm{hr}$ of the procedure and then at 6th, 8th, 12th and 24th hr. The pain score was assessed during a quiet breathing period or at rest (static NRS) and after voluntary cough/ movement (dynamic NRS). The time to the first request and total analgesic consumption of each patient was recorded from the chart. At the times of pain evaluation, the heart rate, the mean arterial blood pressure, respiratory rate and SPO2 was assessed. Any postoperative adverse events such as nausea, vomiting and shivering were recorded and informed to the clinicians on duty for treatment

For the purpose of this study, the following definitions were used:

Time to first analgesia request

a time in minutes (hour) from the end of surgery to a first time analgesia (Diclofenac or tramadol or both) were given.

Total post-operative analgesia consumption

total dose and type of analgesic medication given in $\mathrm{mg}$ within the first 24 hour starting from admission to recovery room.

Post-operative nausea and vomiting 
when a patients experience at least one episode of either nausea or vomiting within 24 hours Shivering

in voluntary repetitive activity of skeletal muscle

Failed TAP block

when the NRS score is $\geq 4$ at 4 th hour post procedure.

Lost follow up

any follow up followed for less than 24 hour for any reason.

Sample size and sampling technique

The sample size was calculated from the data of the primary outcome in a preceding pilot study using all outcome variables. Time to first analgesic request was used to estimate the sample size because it gave us the largest sample size.A priori power analysis for a one-way ANOVA with 3 groups was conducted in $\mathrm{G}^{*}$ Power (using version 3.1.9.2) to determine sample size using an alpha $=0.05$, a power of 0.80 . In order to ensure a minimum of 78 patients needed, an additional 9 patients $(\approx 10 \%)$ were added as attrition rate.

From situational analysis about 130 patients are estimated to take TAP block with dexamethasone or alone during the study period in the two hospitals. Proportional allocation to size (PAS) were determined from these 130 patients, 87 participants were recruited with the probability of about $66 \%$. By considering the consecutive TAP block with dexamethasone or alone, data collection was made on 2 patients for every 3 patients who underwent elective CS under spinal anesthesia and took TAP block with dexamethasone and alone. From number 1 to 3 , one number was selected by lottery method which was used for exclusion for consecutive patient in all groups until the required sample size is reached.

Data processing and analysis

Data were entered and analyzed by SPSS Version 20. The data were tested for normality using histogram and Shapiro-Wilk normality test and homogeneity of variance by Levene's test. Normally distributed and continuous data were analyzed using one way analysis of variance (ANOVA) with post hoc analysis for multiple tests and non-normally distributed data were analyzed using kuruska-walih $\mathrm{H}$ rank test with pairwise comparison. Time to first analgesic request was analyzed using Life table, log rank KaplanMeier survival curves.

The comparison of categorical variable was analyzed using Pearson chi-square test. Data were presented as mean \pm SD for normally distributed, median \pm IQR (25th-75th percentile) and mean rank for non normally distributed data (decision was made by visual inspection of box plot) and categorical data were presented as numbers and frequencies (percentages). P-values $<0.05$ were considered statistically significant.

\section{Results}

\subsection{Demographic and perioperative Characteristics}


Eighty seven (87) patients participated in this study based on whether they received TAP-IVD (transversus abdominis plane block with intravenous dexamethasone) or TAP-PD (transversus abdominis plane block with perineural dexamethasone) at the end of surgery for postoperative analgesia as exposed group and those with TAP (transversus abdominis plane block) alone during postoperative period as control group. There was no significant difference among the three groups with regard to age, BMI, operation duration, Parity, indication for surgery, baseline MAP and HR ( $p$ value $>0.05)$. Previous cesarean section scar was the commonest indication for CS but there is no statistical difference between three groups as depicted in Table 1.

\subsection{Comparison of time to first analgesia request}

Kaplan-Meier curve for the first analgesic request with the patient not receiving any analgesics after $24 \mathrm{~h}$ censored to the right presented in Fig. 2

TAP-PD and TAP-IVD groups had a median time to first analgesic request of $9 \mathrm{hr}(95 \% \mathrm{Cl}, 8-10)$ and $7.5 \mathrm{~h}$ $(95 \% \mathrm{Cl}, 6.5-8.5)$ respectively, which were longer than the TAP alone group $5 \mathrm{hr}(95 \% \mathrm{Cl}, 4.4-5.6)$. A log rank test revealed that the survival distributions for the three groups were statistically significantly different, $\chi^{2}(2)=19.789, p<.0005$.

Pairwise log rank comparisons with a Bonferroni correction shows there was a statistically significant difference in survival distributions for TAP -IVD vs TAP alone, $\chi^{2}(1)=9.39, p=.002$, and TAP-PD vs TAP alone, $\chi^{2}(1)=15.705, p<.0005$. However, the survival distributions for TAP-PD vs TAP -IVD were not statistically significantly different, $\chi^{2}(1)=1.427, p=.235$

Life table shows cumulative proportion of patient not requesting analgesia within $6 \mathrm{hr}$ after admission to recovery in TAP alone group was $28 \%$ compared to $73 \%$ and $83 \%$ in TAP-IVD and TAP-PD group respectively.

\subsection{Comparison of Postoperative Numeric Pain Rating scale at Rest and coughing}

A Kruskal-Wallis test was conducted to determine if there were differences in NRS score between the groups: TAP alone $(n=29)$, TAP-IVD $(n=29)$, TAP-PD $(n=29)$ and it revealed that the difference in NRS scores was not statistically significant at 4 th, 8 th and 12th hours $(p>0.05)$ between three groups at rest. The mean ranks of NRS scores were statistically significantly different between groups at 6 th $(\chi 2(2)=$ $17.5, \mathrm{p}<0.0001)$ and 24th $\mathrm{hr}(\chi 2(2)=39.7, \mathrm{p}<0$.0001). Subsequently, post hoc analysis was made and it revealed statistically significant differences in NRS scores between TAP- PD (mean rank $=35.47$ ) and TAP alone (mean rank = 59.48) $(p=.001)$ and TAP-IVD (mean rank $=37.05)$ and TAP alone $(p=.001)$ groups at 6 th $\mathrm{hr}$ at rest, but not between TAP-PD and TAP- IVD groups. Additionally, post hoc analysis revealed a statistically significant difference between TAP- PD and TAP alone, TAP- PD and TAP-IVD and TAP- IVD and TAP alone with adjusted $p$ value $=0.014,0.0001$ and 0.002 respectively at 24 th at rest. 
NRS scores of TAP-PD group were lower than TAP-IVD at all time during 24 hour at rest but, these were not statistically significant except at 24th hour with adjusted $p$ value of $<0.0001$ Fig. 2 .

The Kruskal Wallis test revealed that the median NRS score were not significant at 4th, 8th and 12th hours $(p>0.05)$ between three groups at coughing. The NRS score were significantly different between the groups at $6^{\text {th }}(\chi 2(2)=31.04, p<.0001)$ and 24 th $h r(\chi 2(2)=36.36, p<.0001)$ post -operatively on coughing. Post hoc analysis shows significant reduction in NRS score between TAP-PD and TAP alone, TAP-IVD and TAP alone at 6 th hour on coughing with adjusted $p$ value $<0.0001$ in both cases, but not between TAP-PD and TAP- IVD groups $(p>0.05)$. Significant difference in NRS score was also seen in all pairwise comparison groups at 24th hour on coughing.

NRS scores of TAP-PD group were lower than TAP-IVD at all time during 24 hour but, these were not statistically significant except at 24th hour with adjusted $p$ value of $<0.0001$ Fig. 3 .

\subsection{Comparison of cumulative analgesia consumption between groups}

A Kruskal-Wallis test revealed total tramadol and Diclofenac consumption were significantly different between groups $\chi 2(2)=25.06, p<.0001$ and $\chi 2(2)=19.93, p=, 0001$ respectively. Pairwise comparison (Post hoc analysis) shows significant difference in median total tramadol consumption between TAP-PD $($ median $=50)$ vs TAP alone $($ median $=100)(p<0.0001)$ and TAP-IVD $($ median $=50)$ vs TAP alone $(p<$ $0.0001)$ but not between TAP- PD and TAP- IVD groups $(p=1)$. It also showed significant reduction in median (IQR) total Diclofenac consumption between TAP-PD (75(0-75)) vs TAP alone $(75(75-150))(\mathrm{p}<$ $0.0001)$ and TAP-IVD $(75(75))$ vs TAP alone $(P<0.0001)$ but not between TAP- PD and TAP- IVD groups $(p=0.186)$ (table 2)

\subsection{Incidence of postoperative complications}

The incidence of nausea over 24 hours was $30 \%$. The proportions of patients with nausea was higher (48\%) In TAP alone group compared to TAP-IVD (20.7\%) and TAP-PD (20.7\%) group, $X^{2}(2, N=87)=7.021, p=0.03, \varphi_{c}=0.28$. But not between TAP-IVD and TAP-PD groups. The rate of vomiting was comparable in all the three groups $X^{2}(2, N=87)=1.58, p=0.453$. The incidence of post operative shivering was (27.5\%). The proportion of post-operative shivering was lower in TAP-PD (18.5\%) and TAP-IVD (17.2\%) when compared to TAP alone (44.8\%), $X^{2}=3.867, p=0.038, \varphi_{c=0.28}$. (Fig. 4)

\section{Discussion}

In our study, TAP-PD and TAP-IVD significantly decreased postoperative pain, reduced total analgesic consumption and prolonged the median time to first analgesic request in postoperative period after elective cesarean section under spinal anesthesia. 
We found that TAP-PD and TAP-IVD provide comparable level of median time to first analgesic request $9 \mathrm{hr}(95 \% \mathrm{Cl}, 8.023-9.977)$ and $7.5 \mathrm{~h}(95 \% \mathrm{Cl}, 6.511-8.489)$ respectively which were significantly higher than TAP alone $5 \mathrm{hr}(95 \% \mathrm{Cl}, 4.414-5.586)$ with $\mathrm{p}$ value of $<.0005$.

Similar to our finding, another RCT by Fouad et al evaluating efficacy of dexamethasone added to bupivacaine in ultrasound guided transversus abdominis plane block showed that time to first analgesic request (TFA) was significantly longer in the dexamethasone group when compared to control (TAP alone) group (438.2 \pm 24.95 min vs. $272.04 \pm 37.51 \mathrm{~min}, \mathrm{P}=0.002)$ (10). In agreement with our finding Uma Datt Sharma(11) also studied the effectiveness of perineural dexamethasone as adjuvant to ropivacaine on transversus abdominis plane block showed that the dexamethasone group showed statistically significant prolongation in time to first analgesic request when compared to the control (TAP alone ) group with a median time of (547.5 vs 387.5, $p<0.001$.

A prospective cohort study by Molla $Y$ and his colleagues in Gondar, Ethiopia also evaluated the effectiveness of transversus abdominis plane block after abdominal surgery and showed the median ( IQR) time to first analgesic request for TAP block was 360(500) minutes, which was comparable with our finding(12).

Furthermore, another study also showed perineural and intravenous dexamethasone has equivalent analgesic benefits and similar safety profiles when used an adjuvant to peripheral nerve block (13). Rosenfeld et al (8) also showed only there was a mean $1.3 \mathrm{hr}$ difference between intravenous and perineural dexamethasone in terms of time to first analgesic request, which is comparable with our study which showed only median $1.5 \mathrm{hr}$ difference.

In contrary to our finding Heesen et al that there was $241(95 \% \mathrm{Cl}, 87-394)$ minute prolongation in TFA in perineural dexamethasone when compared to intravenous dexamethasone in peripheral nerve blocks(14). This discrepancy could be due to a difference in study design, variability in population and surgical procedures (their study included upper and lower extremity blocks).

Our study showed statistically significant differences in NRS scores between TAP- PD and TAP alone $(p<0.05)$ and TAP-IVD and TAP alone $(p<0.05)$ groups at $6^{\text {th }}$ and $24^{\text {th }} \mathrm{hr}$ at rest and on coughing. There was no statistically significant difference in NRS score between TAP-PD and TAP- IVD group at all time during 24 hours except at $24^{\text {th }}$ hour with adjusted $p$ value of $<0.0001$. This is in line with a study by Mamatha Raghukumar et al(15) which showed a higher VAS score in TAP alone and lower VAS score in TAP with perineural dexamethasone groups at $24^{\text {th }}$ hour post operatively after cesarean delivery. In agreement with our finding Kertalov $A$ et al also studied the effect of adding dexamethasone as a ropivacaine adjuvant in ultrasound guided transversus abdominis plane block showed that the there is a statistically significant difference in median VAS score between TAP with perineural dexamethasone and TAP alone group at $6^{\text {th }}(1$ vs $2, p<0.001)$ and $24^{\text {th }}$ hours $(1$ vs $2, p<0.001)$ respectively (16) 
In contrast to our study a study by Fouad et al revealed that there was no difference in VAS score between TAP-alone and TAP with perineural dexamethasone group at $24^{\text {th }} \mathrm{hr}$ both at rest and movement after inguinal herniorraphy (10). This can be due to difference in surgical procedure, design and follow up interval. Furthermore, another study in Egypt showed addition of dexamethasone to bupivacaine for transversus abdominis plane block significantly decreased VAS score at $2 \mathrm{hr}(4.9 \mathrm{vs} .28 .1, \mathrm{P}=0.01), 4 \mathrm{hr}$ (12.2 vs. $31.1, \mathrm{P}=0.01)$ and $12 \mathrm{hr}(15.7$ vs. $25.4, \mathrm{P}=0.02)$ but not at $24^{\text {th }} \mathrm{hr}(\mathrm{p}=0.41)(17)$. This can be due to different dose of bupivacaine (they used $20 \mathrm{ml}$ only) and difference in population and study design

Our study found a significantly reduced post-operative analgesic consumption (tramadol and Diclofenac) in both dexamethasone groups when compared to the control (TAP alone) group.

In agreement with our find a RCT by Sachdeva and Sinha on 70 patients investigated the effect of dexamethasone as additive to Ropivacaine on ultrasound-Guided Transversus abdominis plane block in Cesarean Section, and showed decreased tramadol requirement postoperatively in dexamethasone when compared to the non dexamethasone group (100.00 \pm 0.00 vs. $140.00 \pm 50.26 \mathrm{mg}, \mathrm{P}=0.046)(18)$

Ammar and mahmoud (17) also studied the effect of adding dexamethasone to bupivacaine on TAP block and showed that the total post operative $24 \mathrm{hr}$ morphine consumption was significantly reduced in dexamethasone group 19.2 (8.1-24.2) vs 4.1(1.7-6.2) $p=0.01$. This was comparable with our finding when an opioid conversion factor of morphine to tramadol is applied (1:10). Zhao et al. also showed the two routes of administration (intravenous and perineural) did not show any significant difference in post op analgesic consumption(19).

Desale et al (20) in Asmara, Eritrea conducted a randomized control trial on effectiveness Transversus abdominis plane block after Caesarean section in an area with limited resources showed the mean \pm SD Diclofenac consumption was $87.21 \pm 51.20$. This was comparable with our finding which showed a total Diclofenac consumption of 75 (75-150) in TAP alone group.

Uma Datt Sharma (11) also studied the effectiveness of perineural dexamethasone as adjuvant to ropivacaine on transversus abdominis plane block after spinal anesthesia showed that there was decrement in total tramadol consumption in 24 hour when compared to control group ( $223.33 \pm 56.83$ vs $293.33 \pm 25.7, \mathrm{p}<0.001)$ but, this result is by much higher than our finding, $50(0-100)$ vs100(100-150). This could difference could be justified by difference in population, difference in study design, difference in sample size and surgical procedure (hernia repair vs cesarean section).

Our study showed Incidence of nausea and shivering was significantly lower in the two dexamethasone groups when compared to the non dexamethasone group. This was comparable with a previous study showing significantly lower incidence of nausea in TAP with perineural dexamethasone group when compared to TAP alone(10). This finding is also in agreement with a study showing lower incidence of shivering in patients pretreated with dexamethasone (21)

\section{Abbreviations}


ASA - American Society of Anesthesiologists, BMI -Body Mass Index, CD- Caesarean delivery, IQR-Inter Quartile Range, MI-Milliliters , Mg/kg - Milligram per Kilogram, Msc-Master of Science, NIBP-Non- Invasive Blood Pressure, NRS -Numerical Rating Scale, RCT- Randomized Control Trial, SPSS - Statistical Package for Social Sciences, TAP - Transversus Abdominis Plane Block, TAP-IVD- Transversus Abdominis Plane Block with Intravenous Dexamethasone, TAP-PD-Transversus Abdominis Plane Block with Perineural Dexamethasone, VAS - Visual Analogue Scale

\section{Declarations}

Ethical approval and consent to participate: Ethical approval was secured from Dilla

University institutional review board. Participation in the study was voluntary and based on each patient's ability to give informed consent.

Consent to publish: No individual data.

Availability of data and material: Data is available on request.

Competing interest: None

Funding: This research was funded by Addis Ababa University

Author's contribution: $\boldsymbol{A Z}, \boldsymbol{W A}$, and $B J$ have made substantial contributions to conception, design, analysis, and interpretation of data, participated in the critical review, and editing of the manuscript drafts for scientific merit and depth. HM, SA and SM contributed to conception, design, and acquisition of data, analysis, and interpretation of data as well as on preparing the manuscript to this study.

\section{References}

1. Abebe et al. Factors leading to cesarean section delivery at Felegehiwot referral hospital, Northwest Ethiopia: a retrospective record review. Reprod Health. 2016;13:6.

2. Betrán AP, Ye J, Moller A, Zhang J, Gülmezoglu AM. The Increasing Trend in Caesarean Section Rates: Global , Regional and National Estimates: 1990-2014. PLoS One. 2016;11(2):1-12.

3. Tarekegn SM, Lieberman LS, Giedraitis V. Determinants of maternal health service utilization in Ethiopia: analysis of the 2011 Ethiopian Demographic and Health Survey. BMC Pregnancy Childbirth. 2014;14:161.

4. Abdallah FW, Halpern SH, Margarido CB. Transversus abdominis plane block for postoperative analgesia after Caesarean delivery performed under spinal anaesthesia? A systematic review and meta-analysis. Br J Anaesth. 2012;109(5):679-87. 
5. Jæger P, Grevstad U, Sauter AR, Sørensen JK, Dahl JB. Does dexamethasone have a perineural mechanism of action? A paired, blinded, randomized, controlled study in healthy volunteers. $\mathrm{Br} \mathrm{J}$ Anaesth. 2016;117(5):635-41.

6. D BPP, Greengrass RA, Penning DH, Reynolds JD. Iliohypogastric-ilioinguinal peripheral nerve block for post-Cesarean delivery analgesia decreases morphine use but not opioid-related side effects. CAN J ANESTH 2002. 2002;49(7):694-700.

7. Mishriky BM, George RB, Habib AS. Transversus abdominis plane block for analgesia after Cesarean delivery: a systematic review and meta-analysis. Can J Anesth. 2012;59:766-78.

8. Gorlin AW, Spiro JA, Trentman TL. Perineural versus intravenous dexamethasone as adjuncts to local anaesthetic brachial plexus block for shoulder surgery. Anaesth 2016,. 2016;71:380-8.

9. Rahangdale Rohit, Mark C.Kendall, Robert J. McCarthy LT. The Effects of Perineural Versus Intravenous Dexamethasone on Sciatic Nerve Blockade Outcomes: A Randomized, Double-Blind, Placebo-Controlled Study. Anesth Analg. 2014;118(5):1113-9.

10. Fouad et al. Efficacy of preemptive dexamethasone added to bupivacaine in ultrasound guided transversus abdominis plain block for post operative analgesia after inguinal hernioraphy P. Am J Res Commun. 2016;4(5):27-42.

11. uma dat sharma. Effect of addition of dexamethasone to ropivacaine on post-operative analgesia in ultrasonography-guided transversus abdominis plane block for inguinal hernia repair: A prospective, double-blind, randomised controlled trial. Indian J Anaesth. 2018;62(5):371-375.

12. Molla Y, Belayneh T HJ (2015). Trans Abdominis Plan Block Reduce Postoperative Analgesic Requirement and Prolong Time to First Analgesic Request in Abdominal Surgery at University of Gondar Hospital : Prospective Observational Study. J Anesth Crit Care. 2015;2(4):00066.

13. Hussain N. Equivalent analgesic effectiveness between perineural and intravenous dexamethasone as adjuvants for peripheral nerve blockade a systematic review and meta-analysis. Can J Anaesth. 2018;65(2):194-206.

14. Heesen M, Klimek M, Imberger G, Hoeks SE, Rossaint R, Straube S. Co-administration of dexamethasone with peripheral nerve block: intravenous vs perineural application: systematic review , meta-analysis, meta-regression and trial-sequential analysis. $\mathrm{Br} \mathrm{J}$ Anaesth. 2018;120(2):212-27.

15. Raghukumar M, Majigoudar SS. A randomized clinical comparative trial to study the efficacy of adding Clonidine or Dexamethasone to Bupivacaine ( $0.5 \%$ ) in prolonging the duration of post operative analgesia with ultrasound guided TAP block in cesarean delivery. Indian J Clin Anaesth. 2017;4(2):198-201.

16. Andrijan Kartalov et al. Effect of adding dexamethasone as a ropivacaine adjuvant. Sec Med Sci. 2015;3:35-41.

17. Mahmoud KM. Effect of adding dexamethasone to bupivacaine on transversus abdominis plane block for abdominal hysterectomy: A prospective randomized controlled trial. Saudi J Anaesth. 2012;6(3):229-33. 
18. Sachdeva J, Sinha A. Randomized Controlled Trial to Study the Effect of Dexamethasone as Additive to Ropivacaine on Duration of Ultrasound-Guided Transversus Abdominis Plane Block in Cesarean Section. Indian J Pain. 2016;30(3):181-5.

19. Zhao W, Ou X, Liu J, Zhang W. Perineural versus intravenous dexamethasone as an adjuvant in regional anesthesia: a systematic review and meta-analysis. J Pain Res. 2017;10:1529-43.

20. Kahsay DT, Elsholz W, Bahta HZ. transversus abdominis plane block after Caesarean section in an area with limited resources. South African J Anaesth Analg. 2017;23(4):90-5.

21. Vinathi GA, Latha VS. Efficacy and Potency of Dexamethasone In Comparison With Ketamine and Tramadol in the Prevention of Post-Operative Shivering. IOSR J Dent Med Sci. 2018;17(4):66-72.

\section{Tables}


Table 1socio demographic and perioperative characteristics of patients who underwent elective cesarean section

\begin{tabular}{|c|c|c|c|c|}
\hline & TAP alone & TAP-IVD & TAP - PD & P-Value \\
\hline Age(year) & $29.3 \pm 4.2$ & $29.7 \pm 4$ & $29.5 \pm 4.5$ & 0.951 \\
\hline $\mathrm{BMI}(\mathrm{kg} / \mathrm{m} 2)$ & $26 \pm 1.2$ & $25.8 \pm 1.2$ & $26 \pm 1.2$ & 0.745 \\
\hline Duration of surgery (min) & $50 \pm 11.6$ & $47.6 \pm 9.7$ & $48.8 \pm 10.6$ & 0.692 \\
\hline Parity & & & & 0.810 \\
\hline \multirow{2}{*}{ - Nulliparous (n, \%) } & $10(11.5 \%)$ & $10(11.5 \%)$ & $8(9.2 \%)$ & \\
\hline & $19(21.8 \%)$ & $19(21.8 \%$ & $21(24.1)$ & \\
\hline \multicolumn{5}{|l|}{ - Multiparous (n, \%) } \\
\hline \multicolumn{5}{|l|}{ Previous cesarean section } \\
\hline \multirow[b]{2}{*}{ - Yes (n, \%) } & $16(18.4 \%)$ & $15(17.2)$ & $19(21.8 \%)$ & 0.872 \\
\hline & $13(14.9 \%)$ & $14(16.1)$ & $10(11.5 \%)$ & \\
\hline \multicolumn{5}{|l|}{ - $\quad$ No (n, \%) } \\
\hline Indications & & & & 0.885 \\
\hline \multirow[b]{2}{*}{ - Previous CS scar (n, \%) } & $16(18.4 \%)$ & $15(17.2)$ & $19(21.8 \%)$ & \\
\hline & $7(8 \%)$ & $10(11.5 \%)$ & $6(6.9 \%)$ & \\
\hline - $\operatorname{Big}$ baby (n, \%) & $4(4.6 \%)$ & $3(3.4 \%)$ & $3(3.4 \%)$ & \\
\hline - Malpresentations (n, \%) & $2(2.3 \%)$ & $1(1.1 \%)$ & $1(1.1 \%)$ & \\
\hline - Others (n, \%) & & & & \\
\hline
\end{tabular}

Experience

- year 1 Msc student

$\begin{array}{llll}2(2.3 \%)) & 0(0 \%) & 0(0 \%) & 0.193 \\ 26(29.9) & 27(31 \%) & 25(28.7 \%) & \\ 1(1.1 \%) & 2(2.3 \%)) & 4(4.6 \%)\end{array}$

- year 2 Msc student

- Msc

\begin{tabular}{lllll}
\hline Baseline MAP & $95 \pm 1.8$ & $94.9 \pm 1.8$ & $94.6 \pm 1.9$ & 0.679 \\
\hline Baseline HR & $88.2 \pm 1.8$ & $88.6 \pm 2.4$ & $89 \pm 1.9$ & 0.306
\end{tabular}

Value are presented as: Mean \pm SD, Number (\%), One way ANOVA test, chi-square test and p<0.05 is statistically significant. 
Table 2 Cumulative analgesic consumption of participants after TAP alone, TAP-IVD and TAP-PD

\begin{tabular}{|c|c|c|c|c|}
\hline & TAP alone & TAP-IVD & TAP-PD & P-Value \\
\hline \multicolumn{5}{|c|}{ Cumulative analgesic consumption } \\
\hline \multirow{2}{*}{ - Total tramadol in mg } & $100(100-150)$ & $50(0-100)^{+}$ & $50(0-100)^{+}$ & $<0.0001$ \\
\hline & $75(75-150)$ & $75(75)^{+}$ & $75(0-75)^{+}$ & $<0.0001$ \\
\hline
\end{tabular}

${ }^{+} p<0.05$ compared to TAP lone group, Value are presented as median ( IQR), Kruskal-Wallis test with pairwise comparison

Figures

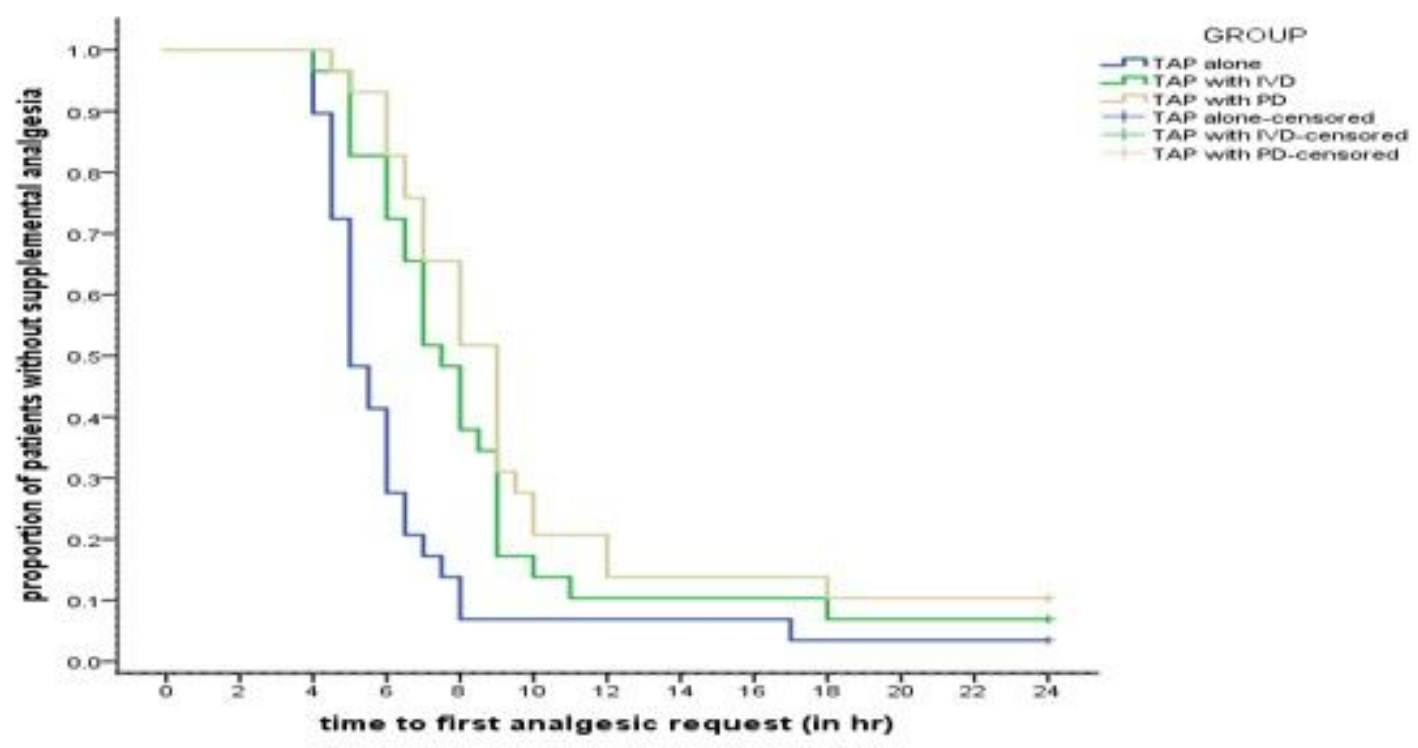

Figure 1 
Kaplan Meier survival plot for time to first analgesic request of participants after TAP alone, TAP-IVD and TAP-PD.

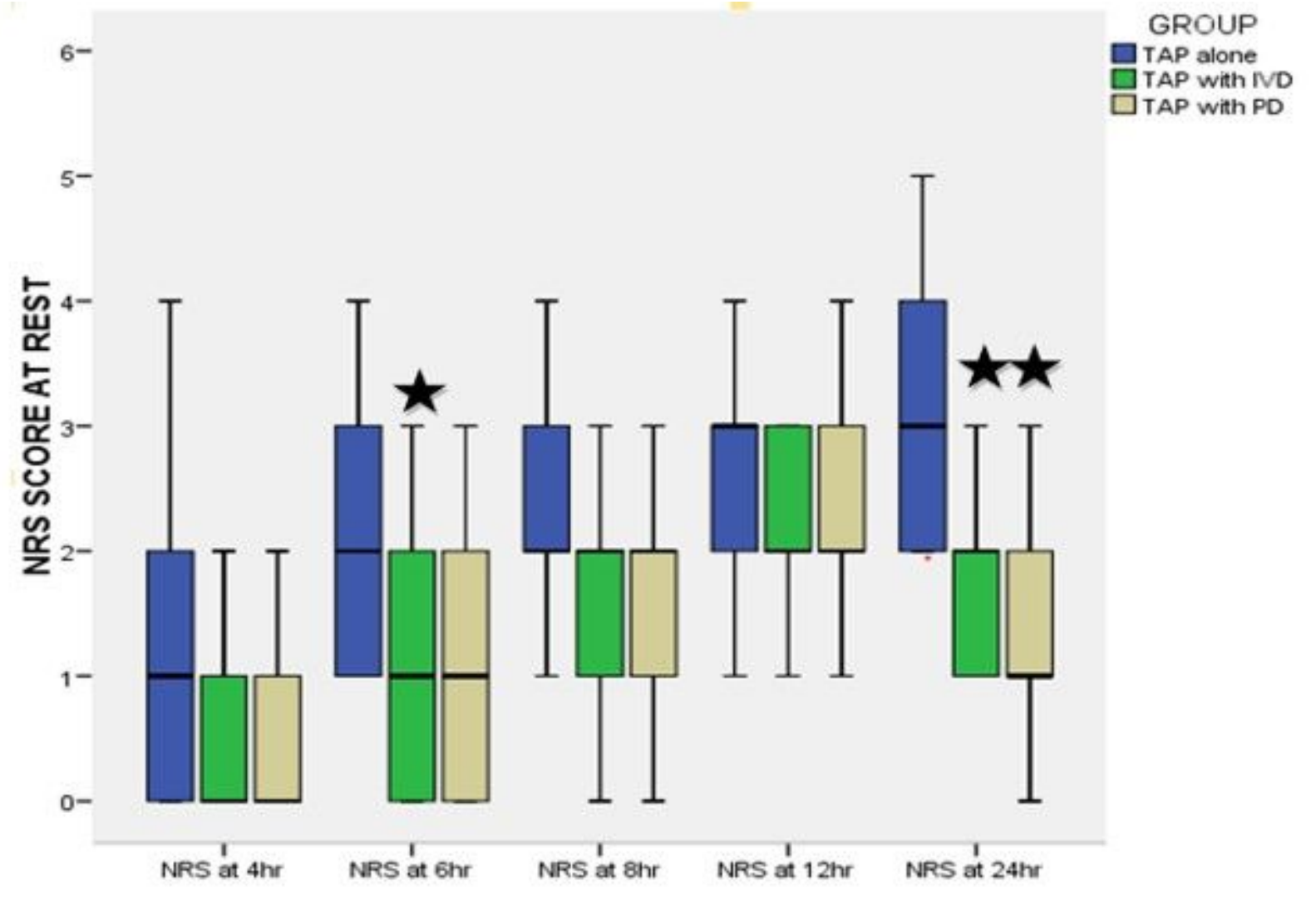

Figure 2

Ip $<0.05$ compared to TAP alone, $\mathbb{Q} \mathrm{p} p<0.05$ between the three groups Comparison of postoperative pain of participants using 11 point NRS score (0-10) at rest. 


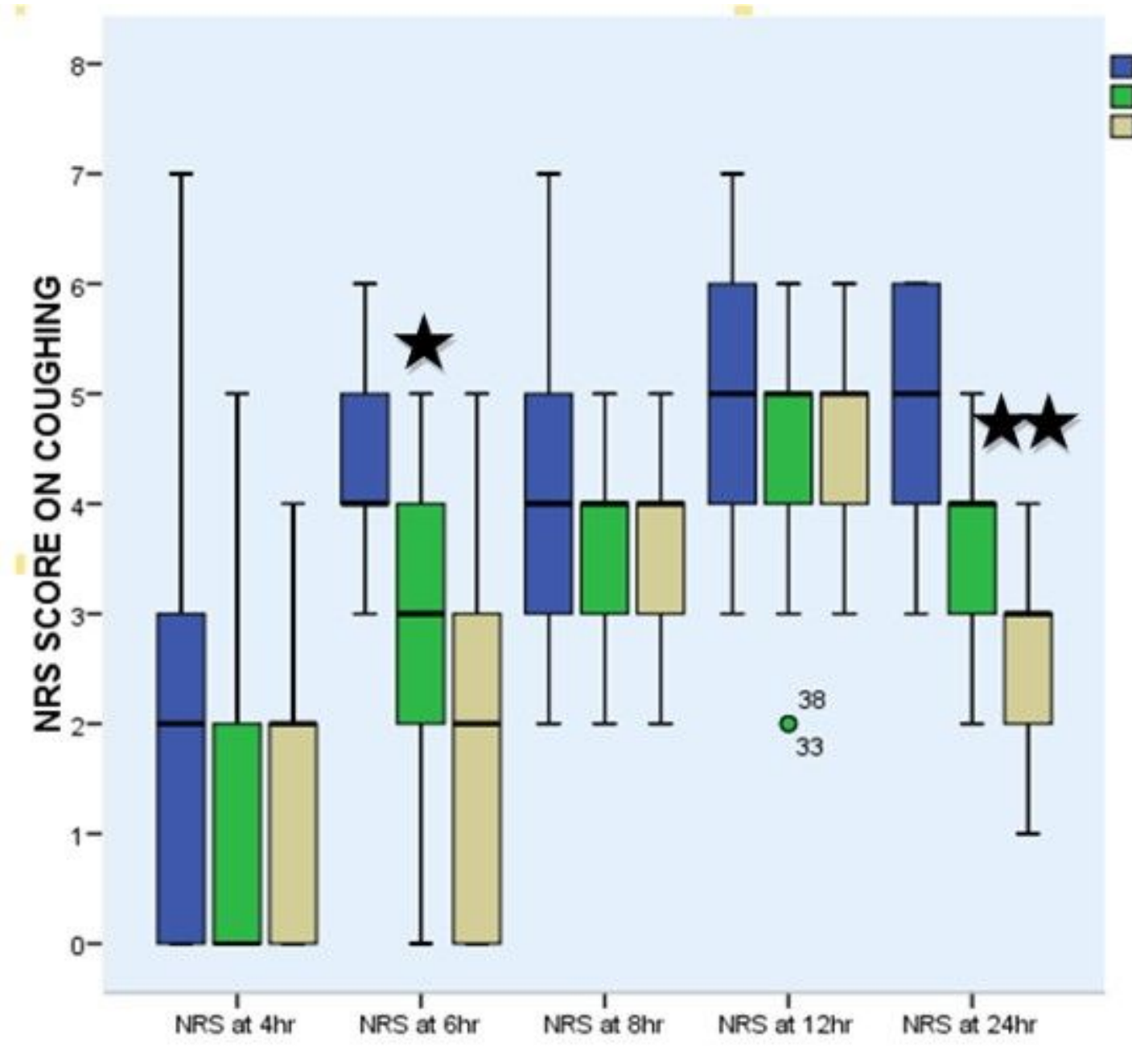

Figure 3

$\square p<0.05$ compared to TAP alone, $\mathbb{Q} \mathrm{p}<<0.05$ between the three groups Comparison of postoperative pain of participants using 11 point NRS score (0-10) at rest. 


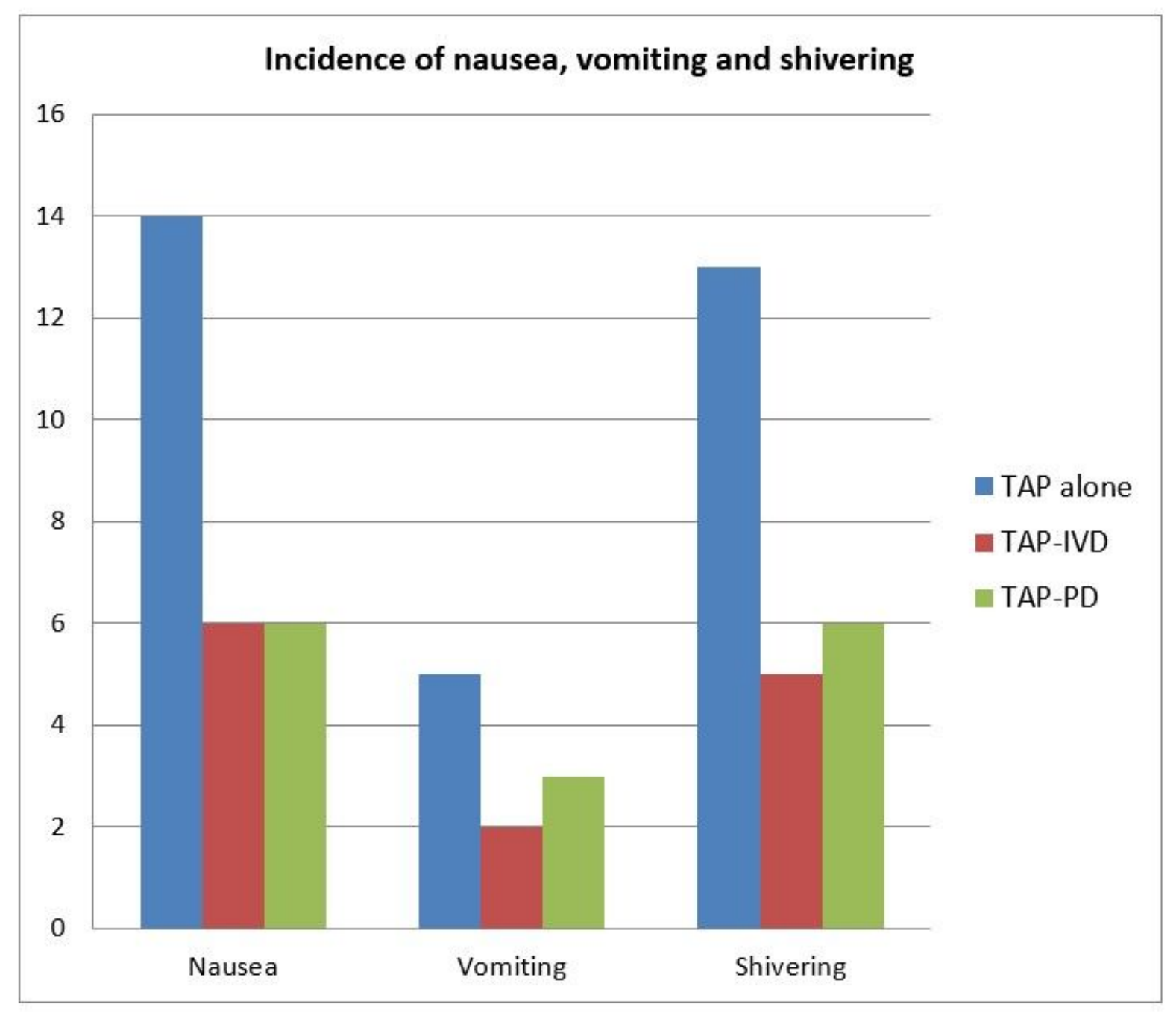

Figure 4

Data are absolute number of patients (Chi-Square Test) incidence of post-operative nausea, vomiting and shivering of participants after TAP alone, TAP-IVD and TAP-PD. 